\title{
The Exterior Square of a Bieberbach Group with Quaternion Point Group of Order Eight
}

\author{
S.A. Mohammad ${ }^{1 *}$, N.H. Sarmin ${ }^{2}$ and H.I. Mat Hassim ${ }^{2}$

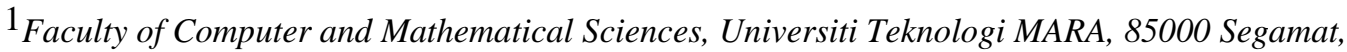 \\ Johor, Malaysia \\ ${ }^{2}$ Department of Mathematical Sciences, Faculty of Science, Universiti Teknologi Malaysia, \\ 81310 UTM Johor Bahru, Johor, Malaysia
}

\begin{abstract}
A Bieberbach group is defined to be a torsion free crystallographic group which is an extension of a free abelian lattice group by a finite point group. This paper aims to determine a mathematical representation of a Bieberbach group with quaternion point group of order eight. Such mathematical representation is the exterior square. Mathematical method from representation theory is used to find the exterior square of this group. The exterior square of this group is found to be nonabelian.
\end{abstract}

Keywords: mathematical structure; exterior square; Bieberbach group; quaternion point group

\section{INTRODUCTION}

Mathematical method from representation theory has been one of the important keys in the study of the structures of a crystal. Bieberbach group is a crystallographic group. In this research, the mathematical representation of a Bieberbach group of dimensions six with quaternion point group of order eight, denoted as $Q_{1}(6)$ is computed. Such mathematical representation is the exterior square of $Q_{1}(6)$, denoted as $Q_{1}(6) \wedge Q_{1}(6)$. In order to compute the mathematical representation of the group, various mathematical approaches have been introduced based on its property.

In 1987, by using the groups' presentation, the nonabelian tensor square of all small groups of order up to 30 were found (Brown et. al., 1987). Meanwhile, the nonabelian tensor squares of 2-generator 2-groups of class 2 using the crossed pairing method were computed in 1999 (Kappe et. al., 1999). The technique of constructing a group $v(G)$ and proving that its subgroup $\left[G, G^{\varphi}\right]$ is isomorphic to the nonabelian tensor square of a group $G$ has been developed (Rocco, 1991), but, due to the limitations of the usage of crossed pairing method, (Blyth \& Morse, 2009) extended the method involving $v(G)$ to compute the nonabelian tensor squares of the polycyclic groups. A research on the mathematical representation of infinite nonabelian 2-generator groups of nilpotency class two has been conducted by using the classification and the nonabelian tensor squares of the groups (Mohd Ali et. al., 2007). Method developed by Blyth and Morse has been used to compute the nonabelian tensor square of Bieberbach groups with abelian point group (Masri, 2009) and also Bieberbach groups with nonabelian point group (Mohd Idrus, 2011).

The objective of this research is to find the mathematical representation of a group which is the exterior square of a Bieberbach group with quaternion point group of order eight.

\section{METHODS}

To find the mathematical representations of a group, the method involving a group $v(G)$ developed by (Rocco, 1991) is used. The group $v(G)$ is defined as follows:

Definition 1 (Rocco, 1991) 
Let $G$ be a group with presentation $\langle G \mid R\rangle$ and let $G^{\varphi}$ be an isomorphic copy of $G$ via the mapping $\varphi: g \rightarrow g^{\varphi}$ for all $g \in G$. The group $v(G)$ is defined to be

$$
\begin{aligned}
v(G) & =\left\langle G, G^{\varphi}\right| R, R^{\varphi},\left[g, h^{\varphi}\right]^{x}=\left[g^{x},\left(h^{x}\right)^{\varphi}\right] \\
& \left.=\left[g, h^{\varphi}\right]^{x \varphi}, \forall x, g, h \in G\right\rangle
\end{aligned}
$$

where $g^{h}=h^{-1} g h$ and $[g, h]=g^{-1} g^{h}$.

The important fact about $v(G)$ is that its subgroup $\left[G, G^{\varphi}\right]$ is actually isomorphic to the nonabelian tensor square of the group $G$. An analysis of the group $v(G)$ for arbitrary finite and infinite groups $G$ as a tool to compute $G \otimes G$ and other mathematical representations has been provided (Blyth \& Morse, 2009). Furthermore, the nonabelian tensor square of a polycyclic group given by a polycyclic presentation can be computed (Eick \& Nickel, 2008). Moreover, if $G$ is polycyclic, then $G \otimes G$ is polycyclic. Hence, $G \otimes G$ has a consistent polycyclic presentation (Blyth \& Morse, 2009). To show that the group is polycyclic, the polycyclic presentation has to be consistent. Thus, the following two definitions are needed.

Definition 2 (Eick \& Nickel, 2008) Polycyclic Presentation Let $F_{n}$ be a free group on generators $g_{i}, \ldots, g_{n}$ and $R$ be a set of relations of group $F_{n}$. The relations of a polycyclic presentation $F_{n} / R$ have the form:

$$
\begin{gathered}
g_{i}^{e_{i}}=g_{i+1}^{x_{i, i+1}} \ldots g_{n}^{x_{i, n}} \text { for } i \in I, \\
g_{j}^{-1} g_{i} g_{j}=g_{j+1}^{y_{i, j, j+1}} \ldots g_{n}^{y_{i, j, n}} \text { for } j<I, \\
g_{j} g_{i} g_{j}^{-1}=g_{j+1}^{z_{i, j, j+1}} \ldots g_{n}^{z_{i, j, n}} \text { for } j<I \text { and } j \notin I
\end{gathered}
$$

for some $I \subseteq\{1, \ldots, n\}$, certain exponents $e_{i} \in \square$ for $i \in I$ and $x_{i, j}, y_{i, i, k}, z_{i, j, k} \in \square$ for all $i, j$ and $k$.

Definition 3 (Eick \& Nickel, 2008) Consistent Polycyclic Presentation
Let $G$ be a group on generated by $g_{1}, \ldots, g_{n}$ and the consistency relations in $G$ can be evaluated in the polycyclic presentation of $G$ using the collection from the left as in the following:

$$
\begin{array}{rlrl}
g_{k}\left(g_{j} g_{i}\right) & =\left(g_{k} g_{j}\right) g_{i} & & \text { for } k>j>i, \\
\left(g_{j}^{e_{j}}\right) g_{i} & =g_{j}^{e_{j}-1}\left(g_{j} g_{i}\right) & & \text { for } j>i, j \in I, \\
g_{j}\left(g_{i}^{e_{i}}\right) & =\left(g_{j} g_{i}\right) g_{i}^{e_{i}-1} & \text { for } j>i, i \in I, \\
\left(g_{i}^{e_{i}}\right) g_{i} & =g_{i}\left(g_{i}^{e_{i}}\right) & \text { for } i \in I, \\
g_{j} & =\left(g_{j} g_{i}^{-1}\right) g_{i} & \text { for } j>i, i \notin I
\end{array}
$$

for some $I \subseteq\{1, \ldots, n\}, e_{i} \in \square$. Then, $G$ is said to be given by a consistent polycyclic presentation.

A Bieberbach group of dimensions six with quaternion point group of order eight that has been considered is isomorphic to the group below:

$$
\begin{aligned}
G & =\left\langle a_{0}, a_{1}, l_{1}, l_{2}, l_{3}, l_{4}, l_{5}, l_{6}\right\rangle \text { where } \\
a_{0} & =\left[\begin{array}{ccccccc}
0 & 0 & 1 & 0 & 0 & 0 & 0 \\
0 & 0 & 0 & 1 & 0 & 0 & 0 \\
-1 & 0 & 0 & 0 & 0 & 0 & 0 \\
0 & -1 & 0 & 0 & 0 & 0 & 0 \\
0 & 0 & 0 & 0 & 1 & 0 & -\frac{1}{4} \\
0 & 0 & 0 & 0 & 0 & 1 & \frac{1}{4} \\
0 & 0 & 0 & 0 & 0 & 0 & 1
\end{array}\right],(1)
\end{aligned}
$$




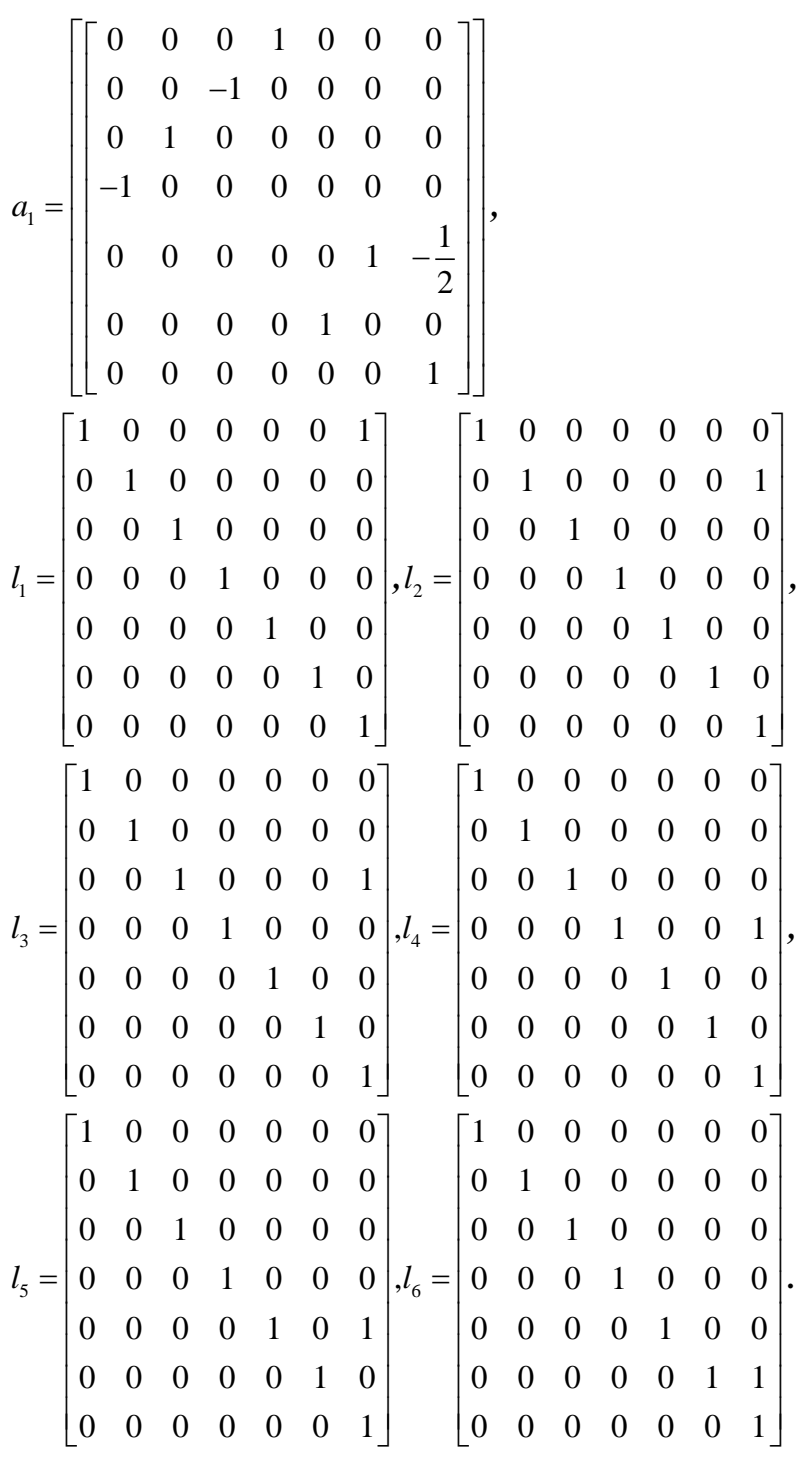

By using matrix form above where $l_{1}, l_{2}, l_{3}, l_{4}, l_{5}, l_{6}$ are its lattices in which its basis matrix is the identity matrix, this group is shown to be isomorphic to a new group which is polycyclic, namely $Q_{1}(6)=\left\langle a, b, c, l_{1}, l_{2}, l_{3}, l_{4}, l_{5}, l_{6}\right\rangle$. A new generator $c$ is developed in the polycyclic presentation so that it satisfies its consistency relations aligning with Definition 3. Thus, the polycyclic presentation can vary depending on what generator $c$ is. Thus, by using all these facts, the $v(G)$ of a Bieberbach group of dimensions six with quaternion point group of order eight is stated as in the following:

\section{Theorem 1}

Let $Q_{1}(6)$ be a Bieberbach group of dimensions six with quaternion point group of order eight as in (1) then, its polycyclic presentation is found to be

$$
\begin{aligned}
Q_{1}(6)= & \left\langle a, b, c, l_{1}, l_{2}, l_{3}, l_{4}, l_{5}, l_{6}\right| \\
& a^{2}=c l_{6}, b^{2}=c l_{5} l_{6}^{-1}, c^{2}=l_{5} l_{6}^{-1}, \\
& b^{a}=b c^{-1} l_{5}^{-1}, c^{a}=c, c^{b}=c, \\
& l_{1}^{a}=l_{3}, l_{1}^{b}=l_{4}, l_{1}^{c}=l_{1}^{-1}, \\
& l_{2}^{a}=l_{4}, l_{2}^{b}=l_{3}^{-1}, l_{2}^{c}=l_{2}^{-1}, \\
& l_{3}^{a}=l_{1}^{-1}, l_{3}^{b}=l_{2}, l_{3}^{c}=l_{3}^{-1}, \\
& l_{4}^{a}=l_{2}^{-1}, l_{4}^{b}=l_{1}^{-1}, l_{4}^{c}=l_{4}^{-1}, \\
& l_{5}^{a}=l_{5}, l_{5}^{b}=l_{6}, l_{5}^{c}=l_{5}, \\
& l_{6}^{a}=l_{6}, l_{6}^{b}=l_{5}, l_{6}^{c}=l_{6}, \\
& l_{j}^{l_{i}}=l_{j}, l_{j}^{l_{i}^{-1}}=l_{j} \\
& \text { for } j>i, 1 \leq i, j \leq 6\rangle .
\end{aligned}
$$

Then, $Q_{1}(6)$ is consistent.

To find the mathematical representation which is the exterior square of a group, $G \wedge G$, the next theorem that indicates $G \wedge G$ is isomorphic to $\left[G, G^{\varphi}\right]_{\tau(G)}$ is stated. Before that, the following definition is needed.

\section{Theorem 2}

Let $Q_{1}(6)$ be a Bieberbach group of dimensions six with quaternion point group of order eight. Then, the central subgroup of $Q_{1}(6)$, denoted as $\nabla\left(Q_{1}(6)\right)$ is given as:

$$
\begin{aligned}
\nabla\left(Q_{1}(6)\right)= & \left\langle a \otimes a, b \otimes b, l_{1} \otimes l_{1},\left(a \otimes l_{1}\right)\left(l_{1} \otimes a\right),\right. \\
& \left.\left(b \otimes l_{1}\right)\left(l_{1} \otimes b\right)\right\rangle .
\end{aligned}
$$

\section{Definition 4 (Blyth \& Morse, 2009)}

Let $G$ be any group. Then $\tau(G)$ is defined to be quotient group $v(G) / \sigma(\nabla(G))$, where

$$
\sigma: G \otimes G \rightarrow\left[G, G^{\varphi}\right] \triangleleft v(G)
$$

defined by $\sigma(g \otimes h)=\rightarrow\left[g, h^{\varphi}\right]$ for all $g, h$ in $G$ is an isomorphism. 
Theorem 3 (Blyth \& Morse, 2009)

Let $G$ be any group. The map $\hat{\sigma}: G \wedge G \rightarrow\left[G, G^{\varphi}\right]_{\tau(G)} \triangleright \tau(G)$ defined $\hat{\sigma}(g \wedge h)=\left[g, h^{\varphi}\right]_{\tau(G)}$ is an isomorphism.

Hence, in this research, $\left[G, G^{\varphi}\right]_{\tau(G)}$ is computed in order to find the exterior square of a group. Since $\tau(G)$ is a subgroup of $\left[g, h^{\varphi}\right]_{\tau(G)}$ coincides with $\left[g, h^{\varphi}\right]$. Therefore, for simplification, $\left[g, h^{\varphi}\right]$ is used instead of $\left[g, h^{\varphi}\right]_{\tau(G)}$

Theorem 4 gives a complete description of the generators of $\left[G, G^{\varphi}\right]$ and $\left[G, G^{\varphi}\right]_{\tau(G)}$ in terms of a polycyclic generating set of $G$. This theorem is used in computing the nonabelian tensor square and the exterior square of a group $G$.

\section{Theorem 4 (Blyth \& Morse, 2009)}

Let $G$ be a polycyclic group with a polycyclic generating sequence $g_{1}, \ldots \ldots, g_{k}$. Then $\left[G, G^{\varphi}\right]$ a subgroup of $v(G)$ is given by

$\left[G, G^{\varphi}\right]=\left\langle\left[g_{i}, g_{i}^{\varphi}\right],\left[g_{i}^{\delta},\left(g_{j}^{\varphi}\right)^{\varepsilon}\right],\left[g_{i}, g_{j}^{\varphi}\right]\left[g_{j}, g_{i}^{\varphi}\right]\right\rangle$

and $\left[G, G^{\varphi}\right]_{\tau(G)}$, a subgroup of $\tau(G)$ is given by

$$
\left[G, G^{\varphi}\right]_{\tau(G)}=\left\langle\left[g_{i}^{\delta},\left(g_{j}^{\varphi}\right)^{\varepsilon}\right],\left[g_{j}^{\varepsilon},\left(g_{i}^{\varphi}\right)^{\delta}\right]\right\rangle
$$

for $1 \leq i<j \leq k$, where

$$
\varepsilon= \begin{cases}1, & \text { if }\left|g_{i}\right|<\infty \\ \pm 1, & \text { if }\left|g_{i}\right|=\infty\end{cases}
$$

and

$$
\delta= \begin{cases}1, & \text { if }\left|g_{j}\right|<\infty \\ \pm 1, & \text { if }\left|g_{j}\right|=\infty\end{cases}
$$

\section{RESULTS AND DISCUSSION}

A mathematical representation, namely the exterior square of $Q_{1}(6) \wedge Q_{1}(6)$, is presented in this section. As mentioned in Section II, the subgroup of $v(G)$, $\left[G, G^{\varphi}\right]_{\tau(G)}$ is isomorphic to the exterior square of a group, therefore the exterior square can be computed by finding its $\left[Q_{1}(6), Q_{1}(6)\right]_{\tau(G)}$. Thus, the exterior square of $Q_{1}(6)$ is presented as follows:

\section{Theorem 5}

The exterior square of $Q_{1}(6)$ is nonabelian and is given as

$$
\begin{aligned}
Q_{1}(6) \wedge Q_{1}(6)=\langle & a \wedge b, a \wedge c, c \wedge a, a \wedge l_{1}, a \wedge l_{2}, l_{2} \wedge a \\
& a \wedge l_{6}, b \wedge l_{1}, b \wedge l_{2}, l_{2} \wedge b, b \wedge l_{5}, l_{5} \wedge b \\
& \left.b \wedge l_{6}, c \wedge l_{2}, c \wedge l_{6}\right\rangle
\end{aligned}
$$

\section{Proof}

The exterior square of $Q_{1}(6)$ is isomorphic to $\left[Q_{1}(6), Q_{1}(6)\right]_{\tau(G)}$ by Theorem 3 . Then, based on Theorem $4,\left[Q_{1}(6), Q_{1}(6)\right]_{\tau(G)}$ is generated by 


$$
\begin{aligned}
& {\left[Q_{1}(6), Q_{1}(6)\right]_{\tau(G)}=} \\
& \left\langle\left[a^{ \pm 1}, b^{ \pm \varphi}\right],\left[b^{ \pm 1}, a^{ \pm \varphi}\right],\left[a^{ \pm 1}, c^{ \pm \varphi}\right],\left[c^{ \pm 1}, a^{ \pm \varphi}\right],\right. \\
& {\left[a^{ \pm 1}, l_{1}^{ \pm \varphi}\right],\left[l_{1}^{ \pm 1}, a^{ \pm \varphi}\right],\left[a^{ \pm 1}, l_{2}^{ \pm \varphi}\right],\left[l_{2}^{ \pm 1}, a^{ \pm \varphi}\right],} \\
& {\left[a^{ \pm 1}, l_{3}^{ \pm \varphi}\right],\left[l_{3}^{ \pm 1}, a^{ \pm \varphi}\right],\left[a^{ \pm 1}, l_{4}^{ \pm \varphi}\right],\left[l_{4}^{ \pm 1}, a^{ \pm \varphi}\right],} \\
& {\left[a^{ \pm 1}, l_{5}^{ \pm \varphi}\right],\left[l_{5}^{ \pm 1}, a^{ \pm \varphi}\right],\left[a^{ \pm 1}, l_{6}^{ \pm \varphi}\right],\left[l_{6}^{ \pm 1}, a^{ \pm \varphi}\right],} \\
& {\left[b^{ \pm 1}, c^{ \pm \varphi}\right],\left[c^{ \pm 1}, b^{ \pm \varphi}\right],\left[b^{ \pm 1}, l_{1}^{ \pm \varphi}\right],\left[l_{1}^{ \pm 1}, b^{ \pm \varphi}\right],} \\
& {\left[b^{ \pm 1}, l_{2}^{ \pm \varphi}\right],\left[l_{2}^{ \pm 1}, b^{ \pm \varphi}\right],\left[b^{ \pm 1}, l_{3}^{ \pm \varphi}\right],\left[l_{3}^{ \pm 1}, b^{ \pm \varphi}\right],} \\
& {\left[b^{ \pm 1}, l_{4}^{ \pm \varphi}\right],\left[l_{4}^{ \pm 1}, b^{ \pm \varphi}\right],\left[b^{ \pm 1}, l_{5}^{ \pm \varphi}\right],\left[l_{5}^{ \pm 1}, b^{ \pm \varphi}\right],} \\
& {\left[b^{ \pm 1}, l_{6}^{ \pm \varphi}\right],\left[l_{6}^{ \pm 1}, b^{ \pm \varphi}\right],\left[c^{ \pm 1}, l_{1}^{ \pm \varphi}\right],\left[l_{1}^{ \pm 1}, c^{ \pm \varphi}\right],} \\
& {\left[c^{ \pm 1}, l_{2}^{ \pm \varphi}\right],\left[l_{2}^{ \pm 1}, c^{ \pm \varphi}\right],\left[c^{ \pm 1}, l_{3}^{ \pm \varphi}\right],\left[l_{3}^{ \pm 1}, c^{ \pm \varphi}\right],} \\
& {\left[c^{ \pm 1}, l_{4}^{ \pm \varphi}\right],\left[l_{4}^{ \pm 1}, c^{ \pm \varphi}\right],\left[c^{ \pm 1}, l_{5}^{ \pm \varphi}\right],\left[l_{5}^{ \pm 1}, c^{ \pm \varphi}\right],} \\
& {\left[c^{ \pm 1}, l_{6}^{ \pm \varphi}\right],\left[l_{6}^{ \pm 1}, c^{ \pm \varphi}\right],\left[l_{1}^{ \pm 1}, l_{2}^{ \pm \varphi}\right],\left[l_{2}^{ \pm 1}, l_{1}^{ \pm \varphi}\right],} \\
& {\left[l_{1}^{ \pm 1}, l_{3}^{ \pm \varphi}\right],\left[l_{3}^{ \pm 1}, l_{1}^{ \pm \varphi}\right],\left[l_{1}^{ \pm 1}, l_{4}^{ \pm \varphi}\right],\left[l_{4}^{ \pm 1}, l_{1}^{ \pm \varphi}\right],} \\
& {\left[l_{1}^{ \pm 1}, l_{5}^{ \pm \varphi}\right],\left[l_{5}^{ \pm 1}, l_{1}^{ \pm \varphi}\right],\left[l_{1}^{ \pm 1}, l_{6}^{ \pm \varphi}\right],\left[l_{6}^{ \pm 1}, l_{1}^{ \pm \varphi}\right],} \\
& {\left[l_{2}^{ \pm 1}, l_{3}^{ \pm \varphi}\right],\left[l_{3}^{ \pm 1}, l_{2}^{ \pm \varphi}\right],\left[l_{2}^{ \pm 1}, l_{4}^{ \pm \varphi}\right],\left[l_{4}^{ \pm 1}, l_{2}^{ \pm \varphi}\right],} \\
& {\left[l_{2}^{ \pm 1}, l_{5}^{ \pm \varphi}\right],\left[l_{5}^{ \pm 1}, l_{2}^{ \pm \varphi}\right],\left[l_{2}^{ \pm 1}, l_{6}^{ \pm \varphi}\right],\left[l_{6}^{ \pm 1}, l_{2}^{ \pm \varphi}\right],} \\
& \left.\left[l_{3}^{ \pm 1}, l_{4}^{ \pm \varphi}, l_{6}^{ \pm \varphi}\right],\left[l_{4}^{ \pm 1}, l_{3}^{ \pm \varphi}\right],\left[l_{3}^{ \pm 1}, l_{3}^{ \pm \varphi}, l_{5}^{ \pm \varphi}\right],\left[l_{4}^{ \pm 1}, l_{5}^{ \pm \varphi}\right],\left[l_{5}^{ \pm 1}, l_{3}^{ \pm \varphi}, l_{4}^{ \pm \varphi}\right],\left[l_{6}^{ \pm \varphi}, l_{4}^{ \pm \varphi}\right],\left[l_{5}^{ \pm 1}, l_{6}^{ \pm \varphi}\right],\left[l_{6}^{ \pm 1}, l_{5}^{ \pm \varphi}\right]\right\rangle \\
& {\left[l^{ \pm},\right.}
\end{aligned}
$$

However, some of the generators can be eliminated since some of them can be written in terms of other generators. First, note that all elements in $\nabla\left(Q_{1}(6)\right)$ are trivial in $Q_{1}(6) \wedge Q_{1}(6)$. Then, by Theorem 2 and 3 , $\left[a, a^{\varphi}\right],\left[b, b^{\varphi}\right],\left[l_{1}, l_{1}^{\varphi}\right],\left[a, b^{\varphi}\right]\left[b, a^{\varphi}\right]$, $\left[a, l_{1}^{\varphi}\right]\left[l_{1}, a^{\varphi}\right]$ and $\left[b, l_{1}^{\varphi}\right]\left[l_{1}, b^{\varphi}\right]$ are trivial in $\left[Q_{1}(6), Q_{1}(6)\right]_{\tau(G)}$.

By using the relations of $Q_{1}(6)$ in (2) and some properties of the commutator calculus, the following results are obtained.
For examples,

$\left[a^{ \pm 1}, b^{ \pm \varphi}\right]:$

$$
\begin{aligned}
{\left[a^{-1}, b^{\varphi}\right] } & =\left[a^{-1},[a, b]^{\varphi}\right]\left[a, b^{\varphi}\right]^{-1} \\
& =\left[a^{-1},\left(c l_{5}\right)^{\varphi}\right]\left[a, b^{\varphi}\right]^{-1} \\
& =\left[a^{-1}, l_{5}^{\varphi}\right]\left[a^{-1}, c^{\varphi}\right]\left[\left[a^{-1}, c\right],\left(l_{5}\right)^{\varphi}\right]\left[a, b^{\varphi}\right]^{-1} \\
& =\left[a^{-1}, l_{5}^{\varphi}\right]\left[a^{-1}, c^{\varphi}\right]\left[a, b^{\varphi}\right]^{-1} \\
& =\left[a, l_{5}^{\varphi}\right]^{-1}\left[a, c^{\varphi}\right]^{-1}\left[a, b^{\varphi}\right]^{-1} \\
& =\left(\left[a, a^{\varphi}\right]^{-2}\left[a, c^{\varphi}\right]^{-1}\right)^{-1}\left[a, c^{\varphi}\right]^{-1}\left[a, b^{\varphi}\right]^{-1} \\
& =\left[a, c^{\varphi}\right]\left[a, a^{\varphi}\right]^{2}\left[a, c^{\varphi}\right]^{-1}\left[a, b^{\varphi}\right]^{-1} \\
& =\left[a, a^{\varphi}\right]^{2}\left[a, b^{\varphi}\right]^{-1} \\
& =\left[a, b^{\varphi}\right]^{-1} .
\end{aligned}
$$

$\left[a, b^{-\varphi}\right]=\left[b^{-1},[a, b]^{\varphi}\right]\left[a, b^{\varphi}\right]^{-1}$

$$
\begin{aligned}
& =\left[b^{-1},\left(c l_{5}\right)^{\varphi}\right]\left[a, b^{\varphi}\right]^{-1} \\
& =\left[b^{-1}, l_{5}^{\varphi}\right]\left[b^{-1}, c^{\varphi}\right]\left[\left[b^{-1}, c\right],\left(l_{5}\right)^{\varphi}\right]\left[a, b^{\varphi}\right]^{-1} \\
& =\left[b^{-1}, l_{5}^{\varphi}\right]\left[b^{-1}, c^{\varphi}\right]\left[a, b^{\varphi}\right]^{-1} \\
& =\left[b, l_{5}^{\varphi}\right]^{-1}\left[b, c^{\varphi}\right]^{-1}\left[a, b^{\varphi}\right]^{-1} \\
& =\left[b, l_{5}^{\varphi}\right]^{-1}\left[b, b^{\varphi}\right]^{-2}\left[a, b^{\varphi}\right]^{-1} \\
& =\left[b, l_{5}^{\varphi}\right]^{-1}\left[a, b^{\varphi}\right]^{-1} \\
& =\left[a, b^{\varphi}\right]^{-1} .
\end{aligned}
$$

$\left[a^{-1}, b^{-\varphi}\right]=\left[a^{-1},[a, b]^{-\varphi}\right]\left[a, b^{-\varphi}\right]^{-1}$

$=\left[a^{-1},\left(c l_{5}\right)^{-\varphi}\right]\left[a, b^{\varphi}\right]$

$=\left[a^{-1}, c^{-\varphi}\right]\left[a^{-1}, l_{5}^{-\varphi}\right]\left[\left[a^{-1}, l_{5}^{-1}\right], c^{-\varphi}\right]\left[a, b^{\varphi}\right]$

$=\left[a, c^{\varphi}\right]\left[a, l_{5}^{\varphi}\right]\left[a, b^{\varphi}\right]$

$=\left[a, c^{\varphi}\right]\left[a, a^{\varphi}\right]^{-2}\left[a, c^{\varphi}\right]^{-1}\left[a, b^{\varphi}\right]$

$=\left[a, a^{\varphi}\right]^{-2}\left[a, b^{\varphi}\right]$

$=\left[a, b^{\varphi}\right]$.

Since $\quad\left[a, b^{\varphi}\right]^{-1}\left[a, b^{\varphi}\right] \quad$ is trivial, then $\left[b, a^{\varphi}\right]=\left[a, b^{\varphi}\right]^{-1}\left[a, b^{\varphi}\right]\left[b, a^{\varphi}\right] . \quad$ However, since $\left[a, b^{\varphi}\right]\left[b, a^{\varphi}\right]$ is trivial in $\left[Q_{1}(6), Q_{1}(6)\right]_{\tau(G)}$, then $\left[b, a^{\varphi}\right]=\left[a, b^{\varphi}\right]^{-1} . \mathrm{Next}$ 


$$
\begin{aligned}
{\left[b^{-1}, a^{\varphi}\right] } & =\left[b^{-1},[b, a]^{\varphi}\right]\left[b, a^{\varphi}\right]^{-1} \\
& =\left[b^{-1},\left(c^{-1} l_{5}^{-1}\right)^{\varphi}\right]\left[b, a^{\varphi}\right]^{-1} \\
& =\left[b^{-1}, l_{5}^{-\varphi}\right]\left[b^{-1}, c^{-\varphi}\right]\left[\left[b^{-1}, c^{1}\right],\left(l_{5}\right)^{-\varphi}\right]\left[b, a^{\varphi}\right]^{-1} \\
& =\left[b^{-1}, l_{5}^{-\varphi}\right]\left[b^{-1}, c^{-\varphi}\right]\left[b, a^{\varphi}\right]^{-1} \\
& =\left[b, l_{5}^{\varphi}\right]\left[b, c^{\varphi}\right]\left[b, a^{\varphi}\right]^{-1} \\
& =\left[b, l_{5}^{\varphi}\right]\left[b, b^{\varphi}\right]^{2}\left[a, b^{\varphi}\right] \\
& =\left[b, l_{5}^{\varphi}\right]\left[a, b^{\varphi}\right] .
\end{aligned}
$$

$\left[b, a^{-\varphi}\right]=\left[a^{-1},[b, a]^{\varphi}\right]\left[b, a^{\varphi}\right]^{-1}$

$=\left[a^{-1},\left(c^{-1} l_{5}^{-1}\right)^{\varphi}\right]\left[b, a^{\varphi}\right]^{-1}$

$=\left[a^{-1}, l_{5}^{-\varphi}\right]\left[a^{-1}, c^{-\varphi}\right]\left[\left[a^{-1}, c^{-1}\right],\left(l_{5}\right)^{-\varphi}\right]\left[b, a^{\varphi}\right]^{-1}$

$$
\begin{aligned}
& {\left[a^{ \pm 1}, c^{ \pm \varphi}\right]=\left[a, c^{\varphi}\right]^{ \pm 1},} \\
& {\left[c^{ \pm 1}, a^{ \pm \varphi}\right]=\left[c, a^{\varphi}\right]^{ \pm 1},} \\
& {\left[a^{ \pm 1}, l_{5}^{ \pm \varphi}\right]=\left[a, l_{5}^{\varphi}\right]^{ \pm 1},} \\
& {\left[l_{5}^{ \pm 1}, a^{ \pm \varphi}\right]=\left[l_{5}, a^{\varphi}\right]^{ \pm 1},} \\
& {\left[a^{ \pm 1}, l_{6}^{ \pm \varphi}\right]=\left[a, l_{6}^{\varphi}\right]^{ \pm 1},} \\
& {\left[l_{6}^{ \pm 1}, a^{ \pm \varphi}\right]=\left[l_{6}, a^{\varphi}\right]^{ \pm 1},} \\
& {\left[b^{ \pm 1}, c^{ \pm \varphi}\right]=\left[b, c^{\varphi}\right]^{ \pm 1},} \\
& {\left[c^{ \pm 1}, b^{ \pm \varphi}\right]=\left[c, b^{\varphi}\right]^{ \pm 1},} \\
& {\left[c^{ \pm 1}, l_{5}^{ \pm \varphi}\right]=\left[c, l_{5}^{\varphi}\right]^{ \pm 1},} \\
& {\left[l_{5}^{ \pm 1}, c^{ \pm \varphi}\right]=\left[l_{5}, c^{\varphi}\right]^{ \pm 1},} \\
& {\left[c^{ \pm 1}, l_{6}^{ \pm \varphi}\right]=\left[c, l_{6}^{\varphi}\right]^{ \pm 1},} \\
& {\left[l_{6}^{ \pm 1}, c^{ \pm \varphi}\right]=\left[l_{6}, c^{\varphi}\right]^{ \pm 1} .}
\end{aligned}
$$$$
=\left[a^{-1}, l_{5}^{-\varphi}\right]\left[a^{-1}, c^{-\varphi}\right]\left[b, a^{\varphi}\right]^{-1}
$$$$
=\left[a, l_{5}^{\varphi}\right]\left[a, c^{\varphi}\right]\left[b, a^{\varphi}\right]^{-1}
$$$$
=\left[a, c^{\varphi}\right]^{-1}\left[a, c^{\varphi}\right]\left[b, a^{\varphi}\right]^{-1}
$$

$\left[b^{-1}, a^{-\varphi}\right]=\left[b^{-1},[b, a]^{-\varphi}\right]\left[b, a^{-\varphi}\right]^{-1}$

$=\left[b^{-1},\left(l_{5}^{-1} c^{-1}\right)^{-\varphi}\right]\left[b, a^{\varphi}\right]$

$=\left[b^{-1},\left(c l_{5}\right)^{\varphi}\right]\left[b, a^{\varphi}\right]$

$=\left[b^{-1}, l_{5}^{\varphi}\right]\left[b^{-1}, c^{\varphi}\right]\left[\left[b^{-1}, c\right], l_{5}^{\varphi}\right]\left[b, a^{\varphi}\right]$

$=\left[b^{-1}, l_{5}^{\varphi}\right]\left[b^{-1}, c^{\varphi}\right]\left[b, a^{\varphi}\right]$

$=\left[b, l_{5}^{\varphi}\right]^{-1}\left[b, c^{\varphi}\right]^{-1}\left[b, a^{\varphi}\right]$

$=\left[b, l_{5}^{\varphi}\right]^{-1}\left[b, b^{\varphi}\right]^{-2}\left[a, b^{\varphi}\right]^{-1}$

$=\left[b, l_{5}^{\varphi}\right]^{-1}\left[a, b^{\varphi}\right]^{-1}$.

By the relations of $Q_{1}(6)$ in (2) $a, b, l_{5}$ and $l_{6}$ commute are also trivial because $l_{i}^{\varphi}=\varphi\left(l_{i}\right)=l_{i}$ and with $c$. Moreover, $l_{5}$ and $l_{6}$ also commute with $a$. Then, $l_{j}^{\varphi}=\varphi\left(l_{j}\right)=l_{j}$. At the end, the remaining generators of $\left[Q_{1}(6), Q_{1}(6)\right]_{\tau(G)}$ are $\quad\left[a, b^{\varphi}\right],\left[a, c^{\varphi}\right],\left[c, a^{\varphi}\right]$, $\left[a, l_{1}^{\varphi}\right],\left[a, l_{2}^{\varphi}\right],\left[l_{2}, a^{\varphi}\right],\left[a, l_{6}^{\varphi}\right],\left[b, l_{1}^{\varphi}\right]$, $\left[b, l_{2}^{\varphi}\right],\left[l_{2}, b^{\varphi}\right],\left[b, l_{5}^{\varphi}\right],\left[l_{5}, b^{\varphi}\right],\left[b, l_{6}^{\varphi}\right],\left[c, l_{2}^{\varphi}\right]$ and $\left[c, l_{6}^{\varphi}\right]$. Then, by Theorem $3, Q_{1}(6) \wedge Q_{1}(6)$ is generated

$a \wedge b, a \wedge c, c \wedge a, a \wedge l_{1}, a \wedge l_{2}, l_{2} \wedge a, a \wedge l_{6}, b \wedge l_{1}$, $b \wedge l_{2}, l_{2} \wedge b, b \wedge l_{5}, l_{5} \wedge b, b \wedge l_{6}, c \wedge l_{2}$ and $c \wedge l_{6}$. The 
next step is to show that $Q_{1}(6) \wedge Q_{1}(6)$ is nonabelian. Since,

$$
\begin{aligned}
{\left[\left[a, b^{\varphi}\right]\left[a, l_{1}^{\varphi}\right]\right] } & =\left[[a, b],\left[a, l_{2}\right]^{\varphi}\right] \\
& =\left[c l_{5},\left(l_{2} l_{4}^{-1}\right)^{\varphi}\right] \\
& =\left[c,\left(l_{2} l_{4}^{-1}\right)^{\varphi}\right]\left[\left[c,\left(l_{2} l_{4}^{-1}\right)\right], l_{5}^{\varphi}\right]\left[l_{5},\left(l_{2} l_{4}^{-1}\right)\right] \\
& =\left[c,\left(l_{2} l_{4}^{-1}\right)^{\varphi}\right]\left[\left[l_{2}^{2} l_{4}^{-2}, l_{5}^{\varphi}\right]\left[l_{5},\left(l_{2} l_{4}^{-1}\right)\right]\right. \\
& =\left[c,\left(l_{2} l_{4}^{-1}\right)^{\varphi}\right] \\
& =\left[c,\left(l_{4}^{-1}\right)^{\varphi}\right]\left[c, l_{2}^{\varphi}\right]\left[\left[c, l_{2}\right], l_{4}^{-\varphi}\right] \\
& =\left[c,\left(l_{4}^{-1}\right)^{\varphi}\right]\left[c, l_{2}^{\varphi}\right]\left[l_{2}^{2}, l_{4}^{-\varphi}\right] \\
& =\left[c, l_{4}^{\varphi}\right]^{-1}\left[c, l_{2}^{\varphi}\right] \\
& =\left(\left[c, l_{2}^{\varphi}\right]^{-1}\right)^{-1}\left[c, l_{2}^{\varphi}\right] \\
& =\left[c, l_{2}^{\varphi}\right]^{2} \\
& \neq 1 .
\end{aligned}
$$

Then, $\left[Q_{1}(6), Q_{1}(6)\right]_{\tau(G)}$ is nonabelian which implies $Q_{1}(6) \wedge Q_{1}(6)$ to be nonabelian.

\section{SUMMARY}

In this paper, the exterior square of a Bieberbach group with quaternion point group of order eight is computed. The result shows that the exterior square of this group is nonabelian.

\section{ACKNOWLEDGEMENTS}

The authors would like to express their appreciation for the support of the sponsor; Ministry of Higher Education (MOHE) Malaysia and Research Management Centre (RMC), Universiti Teknologi Malaysia (UTM) Johor Bahru for the financial funding through the Research University Grant (GUP) Vote No. 13J82. The first author is also indebted to Malaysian Mathematical Sciences Society (PERSAMA) for the sponsorship of fees.

\section{REFERENCES}

Blyth, R. D. \& Morse R. F. 2009, Computing the nonabelian tensor squares of polycyclic groups. Journal of Algebra, 321, 2139-2148.

Brown, R., Johnson, D. L. \& Robertson, E. F. 1987, Some computations of non-abelian tensor products of groups. Journal of Algebra, 111, 177-202.

Eick, B. \& Nickel,W. 2008. Computing Schur multiplicator and tensor square of polycyclic group. Journal of Algebra, 320(1), 927-944.

Kappe, L. C., Sarmin, N. H. \& Visscher, M. P. 1999, Twogenerator two-groups of class two and their nonabelian tensor squares. Glasgow Math., J. 41, 417-430.
Masri, R. 2009, The nonabelian tensor squares of certain Bieberbach groups with cyclic point group of order two. PhD. Thesis, UTM, Malaysia.

Mohd Ali, N. M., Sarmin, N. H. \& Kappe, L. C. 2007, Homological functors of infinite nonabelian 2-generator groups of nilpotency class two - The composition theorem. Proceedings of $5^{\text {th }}$ Asian Mathematical Conference, 612-617.

Mohd Idrus, N. 2011, Bieberbach groups with finite point groups. PhD. Thesis, UTM, Malaysia.

Rocco, N. R. 1991, On a construction related to the nonabelian tensor square of a group. Bol.Soc. Brasil. Mat. (N. S.), 22(1), 63-79. 